\title{
Facetten muslimischen Lebens in Deutschland
}

\author{
Klaus Spenlen
}

„Der Satz, der Islam gehöre zu Deutschland, geht völlig am Empfinden der Deutschen vorbei, sagt der Religionssoziologe Detlef Pollack. Er leitete 2010 eine Umfrage des Exzellenzclusters „Religion und Politik“ an der Universität Münster im Auftrag des Medien- und Sozialforschungsinstituts EMNID. Je 1.000 Menschen ließ er in Ost- und Westdeutschland, in Dänemark, Frankreich, den Niederlanden und Portugal zu ihren Einstellungen zum Islam befragen. „Die Unterschiede zwischen Deutschland und den anderen Ländern sind geradezu dramatisch, wenn es um die persönliche Haltung gegenüber Muslimen geht", so Pollack. Während Niederländer, Franzosen und Dänen mehrheitlich positiv über Muslime denken (zu $62 \%, 56 \%$ und $55 \%$ ), gilt das in Deutschland nur für eine Minderheit von $34 \%$ (West) und $26 \%$ (Ost). Je mehr Kontakte allerdings zu Muslimen bestehen, und je differenzierter die Informationen über sie sind, desto eher werden sie generell positiv gesehen. So geben im Westen etwa $40 \%$ an, zumindest einige Kontakte zu Muslimen zu haben, im Osten nur $16 \%{ }^{1}$

Dieser einleitende Beitrag soll ermöglichen, Hintergrundwissen über Muslime zu vertiefen. Die dazu im Folgenden ausgewählten Facetten sind Teilbereiche gesellschaftlicher Verantwortung, die bei der Frage der Zugehörigkeit des Islam zu Deutschland eine wichtige Rolle spielen. Sie decken aber nicht die komplexe Fragestellung insgesamt ab. Zu der wechselseitigen gesellschaftlichen Verantwortung von Muslimen und Nichtmuslimen für die Entwicklung der Gesellschaft in Deutschland soll deshalb auch nicht den aus unterschiedlichen Wissenschaftsperspektiven verfassten Beiträgen in diesem Sammelband ein weiterer unter neuer Betrachtungsweise vorangestellt, sondern lediglich ein „Einlesen“ in die Fachbeiträge erleichtert werden. Dazu werden Literaturhinwei-

\footnotetext{
${ }^{1}$ Vgl. die 2010 durchgeführte Studie zur religiösen Vielfalt in Europa von Detlef Pollack unter http:// www.uni-muenster.de/Religion-und-Politik/aktuelles/2010/dez/PM_Studie_Religioese_Vielfalt_in_Europa.html sowie die Berichterstattung: EMNID-STUDIE - Mehrheit der Deutschen sieht Muslime negativ, in: ZEIT online vom 01.12.2010, zitiert unter: http://www.zeit.de/gesellschaft/2010-12/islam-studie-toleranz.
} 
se gegeben, die die angesprochenen Themen vertiefen und jeweils nach ihrem Erscheinungsjahr sortiert sind:

Ursula Spuler-Stegemann, Die 101 wichtigsten Fragen Islam, München 2007.

Gisbert Gemein (Hrsg.), Kulturkonflikte - Kulturbegegnungen. Juden, Christen und Muslime in Geschichte und Gegenwart, Bonn 2011.

Nasr Hamid Abu Zaid mit Hilal Sezgin, Der Koran und die Zukunft des Islam, Freiburg 2011.

Nach Angaben der Studie zu Muslimen in Deutschland, die das Bundesamtes für Migration und Flüchtlinge (BAMF) $2009 \mathrm{im}$ Auftrag der Deutschen Islamkonferenz erstellt hat, leben derzeit etwa 3,8-4,3 Millionen Muslime in Deutschland, das sind ca. 4,6-5,2\% der Gesamtbevölkerung. ${ }^{2}$ Diese Muslime leben nicht in einem monolithischen Block „Islam“, denn es gibt allein in Deutschland zwischen 70 und 80 islamische Organisationen und Strömungen verschiedener Schattierungen. ${ }^{3}$

BAMF - Bundesamt für Migration und Flüchtlinge (Hrsg.), Muslimisches Leben in Deutschland. Im Auftrag der Deutschen Islamkonferenz, Nürnberg 2009.

Trotz der Befragung von Personen aus 49 islamisch geprägten Herkunftsländern sind die Angaben des BAMF geschätzte Zahlen, da es die Angaben der telefonisch ca. 6.000 Befragten aus überwiegend islamisch geprägten Staaten sowie Einbürgerungszahlen ehemaliger türkischer Staatsangehöriger samt deren Familienangehörige hochgerechnet hat. Dabei sind Fehler systemimmanent, denn etwa Bulgarien (13,1\% Muslime) sowie Serbien und Montenegro (ca. $21 \%$ ) gehen als „Nicht-muslimisch“ in diejenigen Statistiken oder Umfragen ein, die sich am zentralen Bestimmungsmerkmal „Herkunftsland“ orientieren. Andererseits gibt es in Syrien eine große christliche Population, gleichwohl gilt Syrien als „islamisches Land“. Auch die Zuweisung der türkisch-kurdischen Aleviten zum Islam ist nicht unumstritten. „Es gibt Anhänger der Theorie, dass das Alevitentum ein - wenn auch entfernter - Teil des schiitischen, aber insbesondere auch des in der Türkei vorherrschenden sunnitischen Islam sei; ja dass die Aleviten sogar

\footnotetext{
${ }^{2}$ Vgl. BAMF 2009, S. 11.

${ }^{3}$ Vgl. Volkmar Krech 2009, S. 10 ff.
} 
die besseren Muslime seien. Andere wieder fordern, dass die alevitische Religion in ihrer Eigenheit gesehen werden müsse" ${ }^{4}$ Ebenso werden die türkisch-kurdischen Jesiden herkunftsbezogen-statistisch als Muslime gezählt, obwohl das Jesidentum - als wohl älteste Religion der Welt - nichts mit dem Islam zu tun hat. Und schließlich verstehen sich auch nicht alle Familienangehörigen eines im Rahmen der BAMF-Studie befragten Muslims selbst als Muslim.

Zahlenangaben und Statistiken zur Religionszugehörigkeit sind mit weiteren erheblichen Unsicherheitsfaktoren behaftet:

- In vielen gemischtreligiösen Staaten Asiens, wie z. B. Indien und China, sind die Religionsstatistiken ein Politikum und werden je nach politischen Interessen zugunsten der Mehrheitsreligion oder des Merkmals „religionslos" verändert.

- In den meisten islamisch-geprägten Staaten werden keine offiziellen Statistiken geführt. In islamischen Ländern gilt grundsätzlich die gesamte einheimische Bevölkerung als muslimisch. Zudem gibt es für Willige keine Möglichkeit, sich offiziell vom Islam loszusagen oder „aus dem Islam auszutreten“. Jemand ist Muslim (wie auch Jude) durch die Geburt. Ein muslimischer Vater hat muslimische Kinder (wie andererseits im Judentum die Mutter die Religionszugehörigkeit weitergibt).

- Dort, wo Statistiken - wie in Deutschland - geführt werden, geben diese häufig ein geschöntes Bild. So werden in Deutschland immer noch deutlich mehr Menschen als Mitglieder einer der beiden Großkirchen und damit als "Christen” geführt, obwohl viele von ihnen inzwischen ausgetreten sind. Zudem ist aus soziologischen Untersuchungen zur Religionszugehörigkeit hinlänglich bekannt, dass die formale Zugehörigkeit zu einer Religion nicht viel über den praktizierten Glauben und die tatsächliche Religiosität eines Gläubigen aussagt.

- Es gibt in Deutschland auch keine Pflicht für Muslime, sich mit ihrer Religionszugehörigkeit in ein Register eintragen zu lassen, und das Ausländerzentralregister (AZR) speichert nicht die Religionszugehörigkeit. Solange es dieses nicht gibt, beruhen Zahlen eben auch auf Schätzungen, die von der Herkunftsregion abgeleitet werden.

\footnotetext{
${ }^{4}$ Ursula Spuler-Stegemann 2003, S. 17.
} 
- In diesem Kontext spielt zudem taqîyya eine Rolle. Taqîyya bedeutet „Verstellung“, auch „Vorsicht“ und meint, dass Orientalen aus kulturellen oder religiösen Gründen ihr Wahrheitsverständnis der jeweiligen Situation anpassen. So möchten sie ggf. einem Gast auch dann einen Wunsch nicht abschlagen, wenn er unerfüllbar ist, etwa um ihn nicht zu enttäuschen oder zu kränken. Dies kann dann, wenn die falsche Aussage herauskommt, beim Gast den Eindruck mangelnder Wahrheitsliebe erwecken. Zudem kann ein Muslim in bestimmten Situationen auch z. B. seine religiöse Identität verleugnen, wenn er Nachteile, gar Bedrohung befürchtet. Da taqîyya sich auf den Koran bezieht ${ }^{5}$ und bei Schiiten obligatorisch ist, macht auch dieser Umstand Statistiken über Muslime ungenau, da sie ihre Religionszugehörigkeit bei Bedarf angeben oder verschweigen können, ohne gegen Grundsätze ihrer Religion zu verstoßen.

- In allgemeinen Statistiken wie z.B. dem Spiegel-Almanach oder dem Fischer Weltalmanach liegt die Präferenz von Länderübersichten auf Themen wie Bevölkerung, Wirtschaft, Geographie, Politik. Es scheint so, dass die Religionsstatistik bei Neuauflagen nicht immer angemessen gepflegt wird.

Die meisten Muslime in Deutschland haben einen Migrationshintergrund. Daneben gibt es auch in Deutschland zum Islam konvertierte Deutsche ${ }^{6}$ ohne Migrationshintergrund, deren Zahlen niemand genau kennt ${ }^{7}$, sowie eingebürgerte Muslime. Die immigrierten Muslime stammen ursprünglich aus ca. 40 Ländern. Das Gros von ihnen kommt - in der Reihenfolge der Nennungen aus der Türkei, aus Bosnien-Herzegowina, dem Irak, Marokko, dem Iran, Af-

\footnotetext{
${ }^{5}$ Koranvers 3:28: „Die Gläubigen sollen sich nicht die Ungläubigen anstatt der Gläubigen zu Freunden nehmen. Wer das tut, hat keine Gemeinschaft [mehr] mit Gott. Anders ist es, wenn ihr euch vor ihnen [d.h. den Ungläubigen] wirklich fürchtet. [In diesem Fall seid ihr entschuldigt.] Gott warnt euch vor sich selber. Bei ihm wird es [schließlich alles] enden“. Die Koran-Zitate in diesem Beitrag stammen von Rudi Paret 2004.

${ }^{6}$ Die meisten der zum Islam Konvertierten geben sich dadurch zu erkennen, dass sie ihrem Vornamen einen islamischen Vornamen voranstellen. So wird aus Sven Müller Muhammad Sven Müller. Lediglich dann, wenn der (bisherige) Vorname christlich oder jüdisch konnotiert ist - Christine, David -, besteht zu einem islamischen Vornamen Pflicht.

${ }^{7}$ Das BAMF gibt die Zahl der Konvertiten unter Hinweis auf das Zentralinstitut Islam-Archiv Soest mit 12.000 bis 50.000 an, vgl. BAMF 2007, S. 40.
} 
ghanistan, dem Libanon, Pakistan, Syrien und Tunesien. Sie gehören - wie Angehörige anderer Religionsgemeinschaften auch - den unterschiedlichsten religiösen Richtungen an, sprechen verschiedene Sprachen, haben unterschiedliche Bildungsniveaus, gehören unterschiedlichen sozialen Schichten an und unterscheiden sich hinsichtlich ihrer Aufenthaltsdauer und ihrer Migrationsgründe. Nähere Informationen können entnommen werden:

Sinus Sociovision, Zentrale Ergebnisse der Sinus-Studie über Migranten-

Milieus in Deutschland unter: http://www.sinus-institut.de/uploads/tx_mpdown loadcenter/MigrantenMilieus_Zentrale_Ergebnisse_09122008.pdf (31.08.2012).

Berlin-Institut für Bevölkerung und Entwicklung (Hrsg.), Ungenutzte Potentiale. Zur Lage der Integration in Deutschland, Berlin 2009.

In jedem Fall zeichnen sich Menschen durch multiple Existenzen aus, sie sind eigene Individuen, die nicht-, gemäßigt- oder strengreligiös leben, die keine singulären Identitäten sind und die sich als Projektion für gesellschaftliche Zuordnungen oder die Konstruktion eines gesellschaftsfähigen, berechenbaren Subjekts nicht eignen. Das trifft uneingeschränkt auch für Muslime zu. Mithin verkürzen Religionsmerkmale wie „Muslim“ die Vielheit und Vielfalt der Menschen islamischen Glaubens in unzulässiger Weise. Zudem gewinnt mit Angriffen auf einen Teil ihrer Identität, hier der religiösen, dieser Teil eine übermächtige Bedeutung und kann für manche Muslime, selbst für liberale, so wichtig werden, dass er unter allen Umständen verteidigt werden muss.

Amartya Sen, Die Identitätsfalle. Warum es keinen Krieg der Kulturen gibt, München 2006.

Levent Tezcan, Das muslimische Subjekt: Verfangen im Dialog der Deutschen Islam Konferenz, Konstanz 2012.

Gerade die Religionszugehörigkeit macht deutlich, dass jeder Mensch zufällig in ein geographisches wie religiöses Umfeld hineingeboren wird, mithin mehr oder weniger zufällig als Christ, Jude oder Muslim aufwächst. ${ }^{8}$ Muslime in Deutschland leben überwiegend in den alten Bundesländern, wohl insbesondere deshalb, weil dort eine höhere Nachfrage nach Arbeitskräften besteht:

\footnotetext{
${ }^{8}$ An dieser Stelle kann das ,islamische Konzept von der Natur des Menschen“ ( fitra) ausgespart werden, nach dem jeder Mensch als „Ergebener“ (Muslim) geboren wird, unabhängig von der Religion seiner Eltern.
} 
Tab. 1: Verteilung der Muslime auf die Bundesländer ${ }^{9}$

\begin{tabular}{|c|c|c|}
\hline Bundesland & Muslime in Prozent & Gesamt \\
\hline Baden-Württemberg & 16,6 & \\
\hline Bayern & 13,2 & \\
\hline Berlin & 6,9 & \\
\hline Bremen & 1,6 & \\
\hline Hamburg & 3,5 & \\
\hline Hessen & 10,3 & \\
\hline Niedersachsen & 6,2 & \\
\hline Nordrhein-Westfalen & 33,1 & \\
\hline Rheinland-Pfalz & 4,0 & \\
\hline Saarland & 0,8 & \\
\hline Schleswig-Holstein & 2,1 & $98,4 \%$ \\
\hline Brandenburg & 0,1 & \\
\hline Mecklenburg-Vorpommern & 0,1 & \\
\hline Sachsen & 0,7 & \\
\hline Sachsen-Anhalt & 0,4 & \\
\hline Thüringen & 0,2 & $1,6 \%$ \\
\hline
\end{tabular}

Die durch Lebenswelten (Lebensstil, Wohnung, Kleidung, Mediennutzung (z. B. bevorzugte Fernsehsender, Filme, Zeitungen und Zeitschriften), soziale Kontakte, Freundschaften, Vereinsmitgliedschaften, politische Einstellungen etc.), Wertorientierung und soziale Lage geprägten Lebensauffassungen von Muslimen in Deutschland sind nicht eindimensional auf das Religionsmerkmal zurückzuführen, Muslime sind also nicht als homogene Gruppe zu begreifen. Religion und Herkunft bzw. Zuwanderungsgeschichte beeinflussen zwar ihren Alltag, sind aber letztlich nicht als solitäre identitätsprägende Faktor zu verstehen.

Gleichwohl führen mangelhaft sprachlich-sozialer Austausch mit anderen Milieus, etwa mit nichtmuslimischen und deutschsprachigen, bevorzugter nichtdeutscher Medienkonsum sowie weitere Aspekte, auf die noch eingegangen wird, dazu, dass sich „das Eigene“ in Abgrenzung zum „Fremden“ sieht und Ge-

\footnotetext{
${ }^{9}$ Tabelle 1 wurde entnommen und vom Verfasser neu zusammengestellt: BAMF 2009, S. 107. Für die der Tabelle zugrunde liegende Hochrechnung wandte das BAMF das in den Sozialwissenschaften übliche Verfahren der Berechnung von Konfidenzintervallen an. Das Intervall deckt hierbei den Zahlenbereich ab, in dem der gesuchte Populationswert mit einer vorgegebenen Wahrscheinlichkeit vermutet werden kann, d.h. das Konfidenzintervall hat eine gewisse Breite, die von der Irrtumswahrscheinlichkeit abhängt.
} 
meinsamkeiten eher ausgeblendet werden. Solche Entwicklungen sind zudem Nährboden für wechselseitige Radikalisierungsprozesse.

Brettfeld, Katrin und Wetzels, Peter, Muslime in Deutschland. Integration, Integrationsbarrieren, Religion und Einstellungen zu Demokratie, Rechtsstaat und politisch-religiös motivierter Gewalt. Ergebnisse von Befragungen im Rahmen einer multizentrischen Studie in städtischen Lebensräumen. Im Auftrag des Bundesministeriums des Innern, Berlin 2007.

BMI - Bundesministerium des Innern (Hrsg.), Lebenswelten junger Muslime in Deutschland. Ein sozial- und medienwissenschaftliches System zur Analyse, Bewertung und Prävention islamistischer Radikalisierungsprozesse junger Menschen in Deutschland, Bonn 2011, veröffentlicht 2012.

Religiöse Grundlage für alle Muslime sind Koran und Sunna. Von Muslimen wird der Koran als Wort Gottes, als verbindliche Rechtleitung, Belehrung, Urteilshilfe, praktische Anweisung und als „Zusammenfassung aller religiösen Lehren aller Zeiten“ angesehen. Demnach gilt er als unverfälscht, unveränderbar und ewig gültig. „Er ist die Rechtleitung für alle Menschen und enthält sämtliche Anweisungen und Hilfen, die sie benötigen, um ein Gott gefälliges Leben führen zu können. "10 Als weitere Glaubensquelle gilt die islamische Tradition, die Sunna, die Aussagen Mohammeds sowie Berichte über seine Handlungsweisen in sog. Hadithe-Sammlungen aufführt. Beide Quellen sind für das Leben von Muslimen wichtig, die Hadîthe sind für deren Alltag vielleicht sogar die bedeutendere Grundlage. Gemeinsam bilden sie u. a. die Basis für die „fünf Säulen des Islam“ und können zu konkreten Alltagsfragen - ganz im Sinne eines Katechismus für Katholiken - durch einen Ilmihal ergänzt werden.

Ziele islamischer Religion sind die Generierung von Sinn durch den Islam sowie der Aufbau einer rechtschaffenen islamischen Gesellschaft. Insofern steuert die Religion alle Teilbereiche des Tagesablaufs gläubiger Muslime und soll die Werte Glaube, Verstand, Ehre, Ansehen und Vermögen schützen.

Rudi Paret, Der Koran, Stuttgart 2004, 9. Auflage.

Adel Theodor Khoury (Hrsg.), Der Koran. Erschlossen und kommentiert, Düsseldorf 2006.

\footnotetext{
${ }^{10}$ Sic!, Ursula Spuler-Stegemann 2007, S. 45.
} 
Adel Theodor Khoury (Hrsg.), Der Hadîth. Urkunde der islamischen Tradition, ausgewählt und übersetzt. Band I: Der Glaube, München 2009.

Adel Theodor Khoury (Hrsg.), Der Hadîth. Urkunde der islamischen Tradition, ausgewählt und übersetzt. Band II: Religiöse Grundpflichten und Rechtschaffenheit, München 2009.

Untersuchungen über Religiosität, religiöse Praxis und die Bedeutung der Religion im Alltag von Muslimen in Deutschland konzentrierten sich im Kern bis zum Beginn dieses Jahrtausends auf Aspekte des Betens, des Besuchs von Moscheen sowie auf Einschätzungen der individuellen Bedeutung der Religion. Die Forschung wandte sich erst spät - und kontrovers - dem monotheistischen Religionen inhärenten Alleinstellungsmerkmal zu, das nicht zwischen dem Einen und den vielen Göttern, sondern zwischen „wahr“ und „falsch“ unterscheidet, zwischen Glaube und Unglaube. Damit rückten normative Gebäude, Richtlinien, Dogmen, Lebens- und Heilslehren der „Heiligen Bücher“, in diesem Fall der schariatischen Teile der islamischen Quellen, in den Blick und die mit ihnen verbundenen Energien, die sich gegen eigene Glaubensanhänger, gegen „Häretiker“ und „Ungläubige“ richten. Einerseits übernehmen Religionen damit die klassischen Funktionen von Wertebegründung, Orientierung und Wir-Gefühl. Andererseits erwachsen daraus für den Islam in Deutschland Ablehnungen durch Nichtmuslime, die sich z. B. aus praktiziertem muslimischem Leben ergeben und die zum Teil bis heute ungelöst sind. Dabei geht es um Deutungsmuster des Andersseins, um Fremdheit, um Unterschiede in den Verhaltensweisen und um Fragen nach dem Verständnis gemeinsamer Werte bei kulturellen Unterschieden. Gerade im Alltag erfolgt inzwischen die Bewertung des Islam und seiner Erscheinungs- und Präsentationsformen häufig zentriert im Hinblick auf seine Verträglichkeit mit der Mehrheitsgesellschaft. Ob Muslime integrierbar und integrationsfähig sind, gerät so zu einem Kriterium der Beschreibung von religiöser Heterogenität als tolerierbarer Differenz oder gefährlicher Devianz. Mit diesen Fragestellungen hält auch die Rechtswissenschaft Einzug in die Islamdebatte in Deutschland:

Friedrich-Ebert-Stiftung (Hrsg.), Religion und säkularer Staat Perspektiven eines modernen Religionsgemeinschaftsrechts, in: Policy. Politische Akademie Nr. 20, Berlin 2007. 
Bertelsmann Stiftung (Hrsg.), Religionsmonitor 2008. Muslimische Religiosität in Deutschland. Überblick zu religiösen Einstellungen und Praktiken, Gütersloh 2008.

Mathias Rohe, Das islamische Recht, München 2009.

Markus Gamper, Islamischer Feminismus in Deutschland? Religiosität, Identität und Gender in muslimischen Frauenvereinen, Bielefeld 2011.

Muslime leben in Deutschland in der Diaspora. Sie verarbeiten ihr Fremdsein sowie Erfahrungen in Deutschland oftmals durch Wiederbelebung der mitgebrachten Religion und halten sich an Hergebrachtem und Überliefertem fest, in der Hoffnung, dass diese ihnen ein Gefühl der Sicherheit in Fragen kultureller und erzieherischer Werte vermitteln. Dass eine Transmission eigenkultureller Standards unter Migrationsbedingungen allerdings oftmals keine Hilfe darstellt, zeigt der Konflikt, den gerade junge Muslime erleben, wenn sie ein koordiniertes Handeln zwischen Generationen und Kulturen herzustellen versuchen. Gerade sie müssen in ihrem Alltag in Deutschland erkennbar konkurrierende Anerkennungsmodi synthetisieren, die sich hinsichtlich der kulturellen Codes sowie unterschiedlicher Anforderungen an Rollenverhalten in Familie und Schule/Beruf stark unterscheiden. Zumal leben junge Muslime zwischen zwei Kulturen mit divergierenden Erwartungen, die bei ihnen zu Symptomen einer Identitätskrise führen können. Religionen scheinen hier geradezu auf die Beantwortung der Identitätsfrage spezialisiert zu sein, zumal sie auf die Möglichkeit verweisen können, Kontingenz durch die Akzeptanz ihrer Normen herzustellen und so Komplexität zu reduzieren. Das ist einer der Ansatzpunkte für die islamischen Verbände, aus ihrer institutionellen Dominanz Einfluss auf muslimische Gläubige zu nehmen. Zu nennen sind als größte Dachverbände:

- DİTİB (Diyanet İşleri Türk İslam Birliği - Türkisch-Islamische Union der Anstalt für Religion e. V.) ist mit ca. 150.000 Mitgliedern der größte islamische Verband in Deutschland, untersteht als türkische Behörde unmittelbar der Regierung der Republik Türkei und vertritt mithin den türkisch-nationalen Staatsislam.

- Der Islamrat der Bundesrepublik Deutschland wird von IGMG dominiert, der Islamischen Gemeinschaft Milli Görüş̧ („Nationale Sicht“). Der Islamrat hat insgesamt ca. 50.000 Mitglieder. In ihrem Wappen führt IGMG in ara- 
bischer Sprache den Koranvers 3:19: „Als (einzig wahre) Religion gilt bei Gott der Islam."

- Der Zentralrat der Muslime (ZMD) vertritt mit ca. 12.000 Mitgliedern eine Reihe von türkischen und nicht-türkisch geprägten Mitgliedsvereinen, die z. T. eine strenge Auslegung der Scharia befürworten. ${ }^{11}$

- Der Verband der Islamischen Kulturzentren e. V. (VIKZ) mit knapp 30.000 Mitgliedern steht der Süleymancl-Bewegung nahe und ist streng hierarchisch und zentralistisch organisiert. Der Name des türkischen Dachverbandes des VIKZ, die „Föderation der Vereine zur Förderung der Schüler und Studenten", beschreibt programmatisch die Aktivitäten des VIKZ im Sinne der Süleymancl: Bewahrung der Jugend vor dem Verlust islamischer Glaubenslehre und religiöser Identität in einem nicht-muslimischen Umfeld. Entsprechend betreibt der VIKZ in Deutschland Kinder- und Jugendheime.

Die Verbände unterhalten Moscheen in Deutschland und stellen dazu Imame an. Die gemeinsame zentrale politische Forderung der Verbände ist die Anerkennung des Islam als gleichberechtigte Religionsgemeinschaft in Deutschland, d. h. die Gleichstellung mit den christlichen Kirchen. Dieses wollen sie u. a. durch den Zusammenschluss zum „Koordinierungsrat der Muslime“ (KRM) sowie durch die Anerkennung als Körperschaft des öffentlichen Rechts erlangen. Die Verbände möchten in Deutschland islamische Riten praktizieren, Moscheen und Friedhöfe bauen und islamischen Religionsunterricht in Schulen erteilen. Zu islamischen Organisationen und Dachverbänden sowie zu Imamen und dem islamischen Gemeindeleben in Deutschland geben die folgenden Titel vertiefende Informationen:

Thomas Lemmen, Islamische Vereine und Verbände in Deutschland, in: Friedrich-Ebert-Stiftung (Hrsg.), Gesprächskreis, Migration und Integration, Bonn 2002.

Rauf Ceylan, Die Prediger des Islam. Imame - wer sie sind und was sie wirklich wollen, Bonn 2010.

\footnotetext{
${ }^{11}$ Die hier nicht näher beschriebene Islamische Gemeinschaft in Deutschland e. V. - IGD - kooperiert mit dem ZMD und agiert nicht in jedem Bundesland.
} 
Dirk Halm, Martina Sauer, Jana Schmidt und Anja Stichs, Islamisches Gemeindeleben in Deutschland, in: Bundesamt für Migration und Flüchtlinge, Forschungsbericht 13, im Auftrag der Deutschen Islam Konferenz, Nürnberg 2012.

Politik der Verbände ist es zudem, „den“ Islam in der Öffentlichkeit sichtbar werden zulassen, sei es durch repräsentative Moscheebauten oder Stellungnahmen zu gesellschaftspolitischen Fragen sowie Rechtshilfen bei (medienwirksamen) Konflikten wie dem Tragen islamischer Bedeckungen durch Frauen in Behörden und Schulen, dem Einfordern von Gebetsräumen in Schulen, der Weigerung, an Klassenfahrten oder koedukativem Sportunterricht teilzunehmen, der Anerkennung von islamischen Festtagen als gesetzliche Feiertage, der Rücksichtnahme von Schulen und Berufsfeld im Ramadan u. a. m. ${ }^{12}$

Klaus Spenlen, Schulische Wege zur Integration muslimischer Schülerinnen und Schüler, in: Heiner Barz (Hrsg.), Migration und Bildung. Sozialwissenschaftliche und integrationspolitische Perspektiven, Düsseldorf 2011, S. 4376.

Die Verbände sind auch durch die Moscheegemeinden und Koranschulen an der islamischen Erziehung beteiligt. Weitere Sozialisations- und Erziehungsinstanzen sind Familien sowie Schulen, in denen muslimische Kinder und Jugendliche die Grundlagen ihrer Religion in der „Islamkunde" vermittelt bekamen und die zukünftig durch Islamischen Religionsunterricht ersetzt werden wird.

Der Islam versteht sich als bildungsfreundlich und stellt die Einheit von Glaube und Erziehung heraus (Sure 96, die den sog. Selbstbildungsauftrag („Trag vor im Namen Deines Herrn") enthält sowie der vermeintliche Hadith "Strebe nach Wissen, sei es auch in China"). Ziel der hierarchischen und traditionsorientierten islamischen Erziehung ist idealerweise das ehrerbietige, loyale und gehorsame Familienmitglied, das Eltern und Verwandten sowie älteren Geschwistern Respekt und Folgsamkeit entgegenbringt und die Familie unterstützt. Geschlechts- und Altersrollen sind asymmetrisch angelegt. Das traditionelle Männerbild entspricht einem Patriachat mit starren hierarchischen Strukturen und dem Vater als Hüter und Verteidiger der Familie. Ihm obliegt es, die

\footnotetext{
${ }^{12}$ Vgl. die umfassende und systematische Aufarbeitung religiös-islamischer Konfliktfelder in: Klaus Spenlen 2010, S. 332-388.
} 
Familienehre aufrechtzuerhalten. Sein Ansehen erarbeitet er sich anhand seiner Handlungen, die einen hohen Stellenwert innerhalb der Gemeinschaft einnehmen. Das gesamte gesellschaftliche Leben der Familie bestimmt der Mann, alle Aktivitäten außerhalb des Haushalts und der Familie liegen in seinem Geltungsbereich. Demgegenüber wird der Frau die Rolle als Hausfrau und Mutter zugewiesen. ${ }^{13}$ Sie hat eine niedrigere Rangposition als ihr Mann und wird zu Gehorsam erzogen. Der Erhalt ihrer Ehre und die Einhaltung des strengen Normen- und Wertesystems sind die wichtigsten Säulen ihres Verhaltens. ${ }^{14}$

Die Ehre, die auf Ungleichheit der Geschlechter beruht, wird in manchen Familien als soziale Kapitalform stilisiert. Soziale Diskriminierung aus dem Umfeld ist Grund genug, unter allen Umständen die Ehre wiederherzustellen, um die Achtung und den Respekt der Familie und Gesellschaft zurück zu erlangen. In traditionell-islamischen Familien wird die Ehre eines Mannes als abhängig vom moralischen und respektablen Verhalten seiner weiblichen Familienmitglieder gesehen. Sittsames Verhalten der Frauen begründet die männliche Ehre. Deshalb fällt dem Mann die Aufgabe zu, die Frauen in seiner Familie zu kontrollieren und in der Öffentlichkeit dafür die Verantwortung zu tragen. „Die Ehefrau stellt die größte Gefahr für die Ehre des Mannes dar, da sie es ist, welche die Ehre am nachhaltigsten ruiniert" ${ }^{\text {"15 }}$ und selbst nicht wiederherstellen kann. Die Autoren der folgenden Publikationen haben Einblick in Familien, die traditionellislamisch erziehen:

Halil-Haci Uslucan, Religiöse Werteerziehung in islamischen Familien, erstellt im Auftrag des Bundesministeriums für Familie, Senioren, Frauen und Jugend, Berlin 2008.

Aladin El-Mafaalani und Ahmet Toprak, Muslimische Kinder und Jugendliche in Deutschland. Lebenswelten - Denkmuster - Herausforderungen, in einer Veröffentlichung der Konrad-Adenauer-Stiftung, Sankt Augustin 2011.

Ahmet Toprak, Unsere Ehre ist uns heilig. Muslimische Familien in Deutschland, Freiburg 2012.

\footnotetext{
${ }^{13}$ Vgl. den Hadîth „Das Paradies liegt unter den Füßen der Mütter“, der die Erziehungsrolle der Mütter betont (nach Musnad Ahmad, Sunan An-Nasâ’i, Sunan Ibn Mâjah).

${ }^{14}$ Vgl. Aladin El-Mafaalani und Ahmet Toprak 2011, S. 57 ff. und $41 \mathrm{ff}$.

${ }^{15}$ Norbert Falthauser 2006, S. 81.
} 
Zum Religionsmerkmal „Muslim“ werden gelegentlich von Nichtmuslimen Diskurse über Eugenik und krude Bevölkerungspolitik angestoßen. Als Beispiel mag das meistverkaufte deutschsprachige und „erfolgreichste Hardcover Buch des Jahrzehnts“ gelten, Thilo Sarrazins „Deutschland schafft sich ab“. Da er in diesem Buch von der Erblichkeit der Intelligenz ausgeht und sich damit in die Kritikerriege einreiht, die „dem Islam“ Rückständigkeit unterstellt, ist es notwendig, dies mit Hilfe von Kurzinformationen über Bildungsbeteiligung und Bildungserfolg von Muslimen zu beleuchten. Die wichtigste: Von der vermeintlichen oder tatsächlichen Rückständigkeit des Islam auf eine eingeschränkte Intelligenz von Muslimen zu schließen, ist ähnlich unsinnig wie die Schlussfolgerung zu ziehen, je größer die Einwohnerzahl eines Landes sei, desto höher müsse deren „Brain-output“ sein.

Die einschlägige Fachliteratur fokussiert bei Fragen nach Bildungserfolgen durchweg auf unterschiedliche Gruppen: Migranten allgemein sowie Migranten unterschieden nach Herkunft und/oder Religion. Die jeweiligen Zuordnungen machen es schwierig, in Teilbereichen, in denen Aussagen über die genannten Bevölkerungsgruppen gemacht werden, isolierte Aussagen über Muslime herauszufiltern. Dennoch sind diejenigen Forschungsergebnisse, die Jugendlichen mit Migrationshintergrund geringen Schulerfolg attestieren, hinreichend diskutiert worden. Daher ist bekannt, dass die Hälfte der Schülerinnen und Schüler mit türkischen Wurzeln, also in der Mehrzahl Muslime, in ihren Mathematikund Lesekompetenzen nicht über die niedrigste Kompetenzstufe hinauskommt, im Bereich „Sprachkompetenz“ besonders geringe Leistungen aufweist und lediglich knapp ein Drittel von ihnen im Alltag vorwiegend Deutsch spricht, obwohl sie in Deutschland geboren und zur Schule gegangen sind.

Eine Ursache für geringen Bildungserfolg sind in Erschwernissen und Benachteiligungen von Kindern und Jugendlichen mit Migrationshintergrund durch Lehrkräfte sowie das System Schule zu suchen. Bei der Erklärung unterschiedlicher Bildungserfolge kann zudem das humankapitaltheoretische Modell herangezogen werden. Es erklärt den Bildungserfolg von Kindern und Jugendlichen mit deren familiärer Sozialisation, bei der Eltern ihren Kindern Wissen und Fertigkeiten vermitteln, die den Erfolg im Bildungssystem offensichtlich beeinflussen. Tatsächlich verfügen empirischen Untersuchungen zufolge Migrantenfami- 
lien in Deutschland im Durchschnitt über weniger bildungsrelevante Ressourcen als nicht zugewanderte Familien und sprechen zudem selten Deutsch. Dies gilt insbesondere für Jugendliche, deren Familien aus der Türkei stammen.

Beide Sachverhalte verdeutlichen, dass schulisch nicht reüssierende muslimische Kinder und Jugendliche zwar ggf. durch ihre Eltern vom Bildungserfolg abgehalten werden, dass aber auch die Bildungssysteme Distanz zwischen ihnen und ihren Angeboten aufbauen. Dabei sind sich die meisten Muslime bewusst, dass sich ihre Etablierung in Deutschland wesentlich über eine gute Bildung vollzieht. Viele von ihnen haben deshalb einen ausgeprägten Bildungsoptimismus - trotz struktureller Hürden, Informationsdefiziten und Fehleinschätzungen über das Erreichbare.

Weitere familiengeprägte Werteorientierungen können ebenfalls ursächlich für mangelnden Bildungserfolg sein. Zwar leben türkischstämmige Muslime traditioneller als die Mehrheitsbevölkerung und selbst als Muslime aus anderen Herkunftsländern, zeigen aber kaum Unterschiede zur sonstigen Bevölkerung hinsichtlich gesellschaftlicher Werte wie familiärer Zusammenhalt, Freundschaft, Respekt gegenüber Lebensleistungen, anderen Religionen und Kulturen, Gesetz und Ordnung etc. Diese Ergebnisse sowie die nachfolgenden Literaturhinweise unterstreichen generelle Vorbehalte gegenüber sog. „Ethnic-Marker“:

Deutsches PISA-Konsortium (Hrsg.), PISA 06. Pisa 2006 in Deutschland. Die Kompetenzen der Jugendlichen im dritten Ländervergleich, Münster 2008.

Heike Diefenbach, Kinder und Jugendliche aus Migrantenfamilien im deutschen Bildungssystem. Erklärungen und empirische Befunde, Wiesbaden 2007. Klaus Spenlen, Integration muslimischer Schülerinnen und Schüler. Analyse pädagogischer, politischer und rechtlicher Faktoren, Münster 2010.

Autorengruppe Bildungsberichterstattung im Auftrag der Kultusministerkonferenz und des Bundesministeriums des Innern, Bildung in Deutschland 2012. Ein indikatorengestützter Bericht mit einer Analyse zur kulturellen Bildung im Lebenslauf, Bielefeld 2012.

$\mathrm{Zu}$ den Faktoren Erziehung/Bildung kommt als weitere zentrale Instanz gegenseitiger gesellschaftlicher Verantwortung der Bereich Arbeit/Beruf hinzu. Er ist mit den vorigen durch die Frage, ob und wie Chancengleichheit verwirklicht wird, eng verknüpft zu sein. Und Chancengleichheit realisiert sich unabhängig 
von der sozialen oder ethnischen Herkunft oder dem Religionsmerkmal. Von diesem gesellschaftlichen Ziel scheint Deutschland - nicht nur im Bereich des Arbeitsmarktes - noch weit entfernt zu sein:

Auswertungen der BIBB-Übergangsstudie zeigen: Jugendliche mit Migrationshintergrund haben nach Beendigung der allgemeinbildenden Schule ein ebenso hohes Interesse an einer Berufsausbildung wie einheimische Jugendliche. Dies gilt auch bei einer Differenzierung nach Schulabschlüssen. Bei den angewandten Strategien der Ausbildungsplatzsuche gibt es gleichfalls keine wesentlichen Unterschiede zwischen beiden Gruppen, auch nicht bei Berücksichtigung der schulischen Voraussetzungen. Dennoch sind die Chancen von nichtstudienberechtigten SchulabsolventInnen mit Migrationshintergrund, rasch nach Schulende in eine vollqualifizierende Ausbildung einzumünden - selbst mit den gleichen schulischen Voraussetzungen - wesentlich geringer. Erheblich häufiger durchlaufen SchulabgängerInnen aus Migrantenfamilien daher schwierige und langwierige Übergangsprozesse bei der Suche nach einer beruflichen Ausbildung. ${ }^{16}$

Das bedeutet, dass Migranten, mithin auch ein Teil der Muslime, trotz gleicher formaler Qualifikation sowie vergleichbaren Bemühungen um einen Arbeitsplatz gegenüber anderen Bewerbern durchweg als Verlierer übrigbleiben. Hier kann als ein Grund Diskriminierung aufgrund von Religion oder Herkunft vermutet werden:

Antidiskriminierungsstelle des Bundes (Hrsg.), Benachteiligungserfahrungen von Menschen mit und ohne Migrationshintergrund im Ost-West-Vergleich. Expertise des Sachverständigenrates deutscher Stiftungen für Integration und Migration, Berlin 2012.

Die Universität Konstanz und das Institut zur Zukunft der Arbeit (IZA) bestätigen in einer Studie, dass trotz Antidiskriminierungsgesetz und allgemein wachsender Integrationsbereitschaft Stellenbewerber mit ausländischen Wurzeln auf dem deutschen Arbeitsmarkt noch immer eklatant benachteiligt werden. Dazu hatten sie in einem Feldversuch über tausend Bewerbungen auf Praktikumsstellen für Wirtschaftsstudierende verschickt. Sie verwendeten inhaltlich gleichwertige Bewerbungsunterlagen, denen per Zufall ein Name eindeutig deutscher oder türkischer Herkunft zugeordnet wurde. Die fiktiven Bewerber hatten nicht nur vergleichbare Qualifikationen und Fähigkeiten, sondern waren

\footnotetext{
${ }^{16}$ Sic!, Ursula Beicht und Mona Granato 2009, S. 2.
} 
zudem ausnahmslos deutsche Staatsbürger und Muttersprachler. Das Ergebnis: Bewerber mit türkischen Namen erhielten insgesamt $14 \%$ weniger positive Antworten.

Leo Kaas und Christian Manger, Ethnic Discrimination in Germany's Labour Market: A Field Experiment, in: Forschungsinstitut zur Zukunft der Arbeit / Institute for the Study of Labor, Discussion Paper No. 4741, Konstanz 2010, unter: http://ftp.iza.org/dp4741.pdf.

Bisher wurden außerdem Abschlüsse, die im Ausland erworben worden waren, in Deutschland nicht als gleichwertig mit hier erworbenen Abschlüssen anerkannt. Diese Praxis soll sich mit dem Anerkennungsgesetz vom 1. April 2012 ändern, das offensichtlich dem Arbeitskräftemangel in Deutschland geschuldet ist. Erfahrungen mit dem neuen Gesetz liegen noch nicht vor, weil es erst am 1. Dezember 2012 in Kraft getreten ist.

Dass Frauen, zumal kopftuchtragende Muslima, von all diesen Nachteilen in besonderem Maße betroffen sind, wird ausdrücklich betont.

Zudem wandern jährlich hochqualifizierte Akademiker mit Migrationshintergrund, unter ihnen viele Muslime, aus, weil sie in Deutschland bei Bewerbungen nicht ihren Abschlüssen entsprechend berücksichtigt werden - in anderen Ländern werden sie hingegen umworben. So kommt es dazu, dass z. B. Muslime mit deutschem Pass in die Heimat ihrer Vorfahren auswandern, in der sie nicht geboren wurden und die sie lediglich von Besuchsaufenthalten kennen. Politik und Wirtschaft problematisieren vor dem Hintergrund eines drohenden Fachkräftemangels und der zuletzt rückläufigen Zuwanderung die Auswanderung Hochqualifizierter als „Brain-Drain“. Die folgende Literatur ermöglicht vertiefende Einblicke:

Bettina Englmann, Standards der beruflichen Anerkennung. In: Aus Politik und Zeitgeschichte, H. 44/2009, S. 19-24.

Arnd-Michael Nohl und Anja Weiß, Jenseits der Greencard: Ungesteuerte Migration Hochqualifizierter. In: Aus Politik und Zeitgeschichte, H. 44/2009, S. $12-18$.

Yasar Aydin, Der Diskurs um die Abwanderung Hochqualifizierter türkischer Herkunft in die Türkei, in: Hamburgisches WeltWirtschaftsInstitut (Hrsg.), HWWI Policy Paper 3-9, Hamburg 2010. 
Abschließend wird ein Blick auf den sog. „Arabischen Frühling“ gewagt, der als Vorbote großer Umgestaltungen im Nahen Osten und teilweise auch in auBernahöstlichen Staaten gedeutet werden kann und die nachkoloniale Welt der überwiegend willkürlichen Staatenbildungen zusammenbrechen lassen wird. An dieser Stelle wird er als Beispiel für das Eingebundensein nationaler Gesellschaften in den globalen Austausch via Internet zu politischen und gesellschaftlichen Veränderungen herangezogen. Wie islamisch ist dieser Arabische Frühling? Welche Auswirkungen kann er auf islamische Gemeinschaften, auf die Region, gar auf die Gesellschaft in Deutschland haben?

Zunächst: Die Betonung der Einheit Gottes im Islam ist Motor der Umma, die seit Mohammed als Vorstellung von der Einheit der islamischen Gemeinschaft als Idealbild und Motivation fortbesteht. Das erklärt, weshalb Muslime Anteil nehmen an Entwicklungen von islamischen Gemeinden außerhalb der eigenen Community. Zudem haben sich durch Migrationsströme und technische Vernetzungen transnationale soziale Räume gebildet, auch innerhalb des Islam, sog. virtual communities, die die Ortsbindungen von Gemeinschaften aufheben. Diese Grenzübertritte haben für alle gesellschaftspolitischen Bereiche Konsequenzen.

Informationsgesellschaften zeichnen sich dadurch aus, dass sie gemeinsame transnationale Interessen mit nationalen wie internationalen Akteuren zu lösen versuchen, wobei Führungsriegen auch in politische Entscheidungen der Nationalstaaten eingreifen - und transnational zurückwirken, indem sie aus strategischen Gründen lokal Lösungen erproben, die ggf. andernorts als Blaupausen dienen. Veränderungen wirtschaftlicher, politischer und sozialer Strukturen hier oder andernorts charakterisieren ein Weltsystem, das das Bild gegeneinander abgeschlossener Einzelgesellschaften durch ein Bild ersetzt, in dem Gesellschaften, Kulturen und Individuen miteinander vernetzt sind und darüber voneinander lernen. Neben der Globalisierung findet in einem dialektischen Prozess also zugleich eine Lokalisierung statt. Im Rahmen der Dialektik von Globalisierung und Lokalisierung bestehen Universalismus und Partikularismus nebeneinander und beeinflussen sich gegenseitig. ${ }^{17}$

\footnotetext{
${ }^{17}$ Als Neologismus wird inzwischen dafür der Begriff „Glokalisierung“ verwendet, der Roland Robertson zugerechnet wird.
} 
Das gilt auch, vielleicht in besonderem Maße, für die Umma, die Weltgemeinschaft der Muslime. Was sich also in der arabischen Welt unter dem Label „Arabischer Frühling“ ereignet, interessiert und berührt Muslime weltweit. Deshalb achten auch Muslime in Deutschland sehr genau darauf, wie deutsche oder „westliche" Politiker sich zu den Veränderungen in den Transformationsstaaten der arabischen Welt verhalten. Der Arabische Frühling konnte sprießen, weil sich die dortigen Akteure Glaubwürdigkeit als strukturellen Vorteil gegen bestehende Herrschaftsstrukturen erwerben konnten. Die Veränderungen verdeutlichen daher säkularen und pluralen Gesellschaften die Notwendigkeit, lokal wie global ihre Werte mit ihren Interessen in Einklang zu bringen, um glaubwürdig alternative Gesellschaftskonzepte aufzuzeigen. D. h., Menschenrechte und Grundwerte sind unteilbar, gelten universell und müssen über nationale Grenzen hinaus zur Geltung gebracht werden. Und dies selbst dann, wenn die Gesellschaft mit bis dato „fremden“ Begehren konfrontiert wird oder diese eigenen Interessen zuwiderlaufen. Und Muslime achten auch auf den Sprachgebrauch, der Akteure des Arabischen Frühlings bislang nach Bedarf als Demokraten, gemäBigte/moderate (sic!) Islamisten, Fundamentalisten, Salafisten, Angehörige islamistischer/terroristischer Organisationen u. a.m. und die politischen Entwicklungen wahlweise als Verwirklichung des Selbstbestimmungsrechts der Völker, als demokratische Bewegungen, als Aufstand gegen Despoten, Bürgerkriege, Religionskriege, finale Lösung des Nahostkonflikts usw. bezeichnen. ${ }^{18}$ In jedem Fall scheinen in diesen Regionen religiös-politische Auseinandersetzungen zwischen islamisch-politischen Kontrahenten sowie mit christlichen, jüdischen und weiteren religiösen Minderheiten unausweichlich, und es ist zudem zu erwarten, dass diese Entwicklungen auch das Verhältnis von Muslimen zu Nichtmuslimen in Deutschland beeinflussen und wechselseitigen Irritationen, Vorurteilen und Ablehnungen Vorschub leisten werden.

Wer derzeit (November 2012) die Entwicklungen in Ägypten verfolgt, dem muss klar werden, dass Fortschritt, Modernisierung, Gemeinschaft, Bedürfnisbefriedigung, Teilhabe usw. dort wie in anderen Staaten des sog. Arabischen Frühlings nicht von politischer Theoriebildung oder den Wünschen der Bevöl-

\footnotetext{
${ }^{18}$ Vgl. zum Sprachgebrauch im Kontext von „Islam“ den Beitrag von Nina Kalwa (S. 139-158) in diesem Sammelband.
} 
kerung, sondern durch Beschwörung der ersten islamischen Gemeinschaften unmittelbar aus den islamischen Quellen Koran und Sunna abgeleitet werden. Dafür sorgen allein die parlamentarischen oder strategischen Mehrheiten von Muslimbrüdern, Salafisten und anderen fundamental-islamischen Gruppierungen. Sie alle behaupten, die Probleme der Menschheit könnten gelöst werden, wenn die Welt die Einheit und Einzigkeit Gottes (tauhīd) anerkennen würde. Mithin sind die von westlichen Politikern und einem Teil der Presse verwendeten Zuschreibungen wie Demokratie, Marktwirtschaft, Säkularisierung, Freiheitsbewegung usw. Euphemismen und auf den Arabischen Frühling bezogen unzutreffend. Und mit (Etappen-)Siegen der radikalen Islamisten dort erstarken konservative islamische Kräfte und Organisationen auch in Deutschland.

Informationen sind der einzige Weg, sich mit Ängsten und überzogenen Erwartungen auseinanderzusetzen. Einen aktuellen Einblick zu den gerade erst begonnenen politischen, demografischen, ethnologischen und theologischen Entwicklungen in der arabischen Welt vermitteln:

Emmanuel Todd, Frei! Der arabische Frühling und was er für die Welt bedeutet, München 2011.

Bundeszentrale für politische Bildung (Hrsg.), Arabische Zeitenwende. Aufstand und Revolution in der arabischen Welt. Schriftenreihe Band 1243, Bonn 2012.

Eine entscheidende Frage zur Zukunft Deutschlands wird diejenige sein, wie Muslime hier wahrgenommen werden können und wahrgenommen werden wollen. Werden die Ressourcen von Menschen mit Migrationshintergrund, mithin auch diejenigen vieler Muslime, in Deutschland weiterhin unterschätzt oder anerkannt? Richten sich die Blicke auch zukünftig eher auf Problemlagen und fokussieren sie auf Arbeitslosigkeit, Transferleistungen und Zwangsheirat? Wie können Pauschalierungen aus der Defizitperspektive als Irrtum oder Demagogie enttarnt werden? Was kann die gesamte Gesellschaft gegen einseitige Berichterstattungen in den Medien über Muslime oder "den“ Islam unternehmen, in denen deren Rollen als Opfer oder Täter verfestigt werden? ${ }^{19}$ Was können Schulen, Jugendeinrichtungen und Eltern leisten, um Präferenzen muslimischer

\footnotetext{
${ }^{19}$ Vgl. exemplarisch Sabine Schiffer 2010.
} 
Kinder und Jugendlicher beim Medienkonsum auf deutsche Sender zu richten? Welchen Beitrag an Transparenz können Muslime über sich und ihren Glauben beisteuern? Welche Optionen haben sie, um sich einem Wohnen in ethnisch verdichteten Wohnquartieren zu entziehen? Welche Strategien müssen Muslime entwickeln, um Loyalitätseinforderungen ihrer Community zu Lasten der Gesamtgesellschaft abzuwehren? Welche alternativen Strategien zu „Rückzug in die eigene Community“ und „Extremismus“ stehen Muslimen zur Verfügung, wenn sie Ablehnung und Diskriminierung erfahren? Müssen sich Muslime bei Bedarf und immer wieder neu von extremistischen oder islamistischen Aktivitäten öffentlich distanzieren? Wenn ja: Wer soll sich erklären? Können von demselben Personenkreis bei gesellschaftlichen Krisen regelmäßig Loyalitätsadressen an die Mehrheitsgesellschaft erwartet werden? Warum tolerieren viele Muslime, dass die Dachverbände als ihr Sprachrohr wahrgenommen werden, obschon höchstens $20 \%$ von ihnen Mitglied sind und lediglich - von DİTİB als türkischer Behörde in Deutschland abgesehen - nur ca. 19\% der Muslime die Verbände kennen und sich noch weniger - ca. 3,5\% - durch sie vertreten fühlen ${ }^{20}$ Wie können Zivilgesellschaft, Muslime und Politik die Aufgabe der individuellen Verbindung verschiedener Kulturen hinreichend unterstützen? Durch welche Maßnahmen kann gesellschaftliche Kohäsion, insbesondere nach bestehenden und zu vermittelnden Gemeinsamkeiten verbindlicher Wertvorstellungen, die die Gesellschaft zusammenhalten, hergestellt werden? Können sich Muslime wie Nichtmuslime darauf verständigen, für die in Jahrhunderten erkämpften Grundwerte, insbesondere der negativen und positiven Religionsfreiheit, der Gleichstellung der Geschlechter, der Meinungs- und Pressefreiheit, als indisponible Menschenrechte einzutreten? Was können Muslime wie Nichtmuslime tun, um sich wechselseitig zu akzeptieren und Ängste zu mindern? Wie kann - als gemeinsame Aufgabe - politischer Einfluss ausländischer Regierungen auf Muslime in Deutschland nachdrücklich eingedämmt werden?

Diese sowie weitere Fragen werden in den Fachbeiträgen dieses Sammelbandes aus unterschiedlichen Perspektiven erörtert.

${ }^{20}$ Vgl. BAMF 2009, S. 174 ff. 


\section{Literatur}

BAMF - Bundesamt für Migration und Flüchtlinge (Hrsg.), Muslimisches Leben in Deutschland - religiöse Vorschriften, muslimischer Alltag und Organisationsformen, Nürnberg 2007.

Dass., (Hrsg.), Muslimisches Leben in Deutschland. Im Auftrag der Deutschen Islamkonferenz, Nürnberg 2009.

Beicht, Ursula und Granato, Mona, Übergänge in eine berufliche Ausbildung. Geringere Chancen und schwierige Wege für junge Menschen mit Migrationshintergrund, in: Friedrich-Ebert-Stiftung (Hrsg.), WiSo direkt. Analysen und Konzepte zur Arbeitsund Sozialpolitik, 2009.

Falthauser, Norbert, Falsche Fremdenfreundlichkeit. Islamisches Frauenbild gefährdet europäische Lebensart. Tübingen 2006.

Krech, Volkhard, Islam und Integration - 12 Thesen, in: Friedrich-Ebert-Stiftung (Hrsg.), Policy Nr. 30, Migration - Religion - Integration, Berlin 2009.

Paret, Rudi, Der Koran. Übersetzt, kommentiert und eingeleitet. Digitale Bibliothek Band 46, Berlin 2004.

Reitze, Helmut (Hrsg.), Media Perspektiven Basisdaten. Daten der Mediensituation in Deutschland 2010, Frankfurt/M. 2010.

Schiffer, Sabine, Die Darstellung des Islams in der Presse. Sprache, Bilder, Suggestionen. Eine Auswahl von Techniken und Beispielen, Nürnberg 2004.

Spenlen, Klaus, Integration muslimischer Schülerinnen und Schüler. Analyse pädagogischer, politischer und rechtlicher Faktoren, Münster 2010.

Spuler-Stegemann, Ursula, Gutachten zu den Aleviten. Erstellt im Auftrag des Ministeriums für Schule, Wissenschaft und Forschung des Landes Nordrhein-Westfalen am 21. Juli 2003.

Dies., Die 101 wichtigsten Fragen - Islam, München 2007.

\section{Internetquellen}

Ette, Andreas und Sauer, Lenore, Abschied für immer oder auf Zeit? Internationale Migration hochqualifizierter Deutscher. 2011, in: Forschung und Lehre, 02/2011, unter: www.forschung-und-lehre.de/wordpress/?p=6825 (31.08.2012). 
